\title{
Etude Préliminaire De La Valorisation Des Tourteaux D’hévéa, D’anacarde Et De Pourghère Chez Les Porcs Durant La Gestation Et La Lactation
}

\author{
Kone Gningnini Alain \\ (Assistant, Msc, Ing. Agronome) \\ Kouakou N'Goran David Vincent \\ (Maître-assistant, PhD, Msc, Ing. Agronome) \\ Angbo-Kouakou Cho Euphrasie Monique \\ (Assistant, Msc, Ing. Agronome) \\ Kouame Kouadio Bertin \\ (Assistant, Ing. Agronome) \\ Yeboue François de Paul \\ (Assistant, Msc, Ing. Agronome) \\ ${ }^{1}$ Institut National Polytechnique Félix Houphouët-Boigny, Yamoussoukro, \\ Côte d'Ivoire \\ Kouba Maryline, Professeur \\ (PhD, Ing. Agronome) \\ INRA-Agrocampus Ouest UMR 1348 Physiologie, \\ Environnement et Génétique pour l'Animal et les Systèmes d'Elevage, \\ Saint-Gilles, Rennes, France
}

doi: 10.19044/esj.2016.v12n30p11 URL:http://dx.doi.org/10.19044/esj.2016.v12n30p11

Abstract

The enhancement the cake of cashew kernels downgraded (Anacardium occidentale) (TA), the detoxified cake of kernels rubber (Hevea brasiliensis) (TH) and jatropha (Jatropha curcas) (TJ) in pig feed, was study on 21 pregnant gilts $(73.4 \pm 8.1 \mathrm{~kg})$. These females randomly distributed by group of three, received control diet (RT) and six other diets containing $7.5 \%$ or $15 \%$ of TA (RTA7.5 and RTA15), $7.5 \%$ or $15 \%$ of TH (RTH7.5 and RTH15) or 7.5\% or 15\% of TJ (RTJ7.5 and RTJ15). During the trial of 152 days, gilts under RTJ15 regime and two gilts under RTJ7.5 regime were withdrawn after respectively 14 and 21 days' supply due to a persistent diarrhea. The latest gilt under RTJ7.5 had aborted at 90 days of gestation. No significant difference was observed on the average litter size (9.2 piglets). The average weight of piglets at birth ranged from $1.3 \pm 0.2 \mathrm{~kg}$ (RTA15) and $1.1 \pm 0.2 \mathrm{~kg}$ (RTH7.5), while the average daily gain (ADG) of 
the weaned piglets to 28 days of age fluctuated between $124 \mathrm{~g} / \mathrm{d}$ (RTA15) and $164 \mathrm{~g} / \mathrm{d}$ (RTH7.5). The daily average gain of weaned piglets and RTH7.5 RTH15 diets was higher than those of weaned piglets submitted to RTA7.5 and RTA15 $(\mathrm{P}<0.05)$. Thus, the $\mathrm{TH}$ and the TA could be incorporated to $15 \%$ in the diets of gilts in reproduction..

Keywords: Pigs, cakes, rubber, jatropha, cashew, reproduction

\section{Résumé}

La valorisation du tourteau des amandes déclassées de cajou (Anacardium occidentale) (TA), des tourteaux détoxifiés des amandes d’hévéa (Hevea brasiliensis) (TH) et de pourghère (Jatropha curcas) (TJ) dans l'alimentation des porcs a été étudiée sur 21 cochettes gestantes (73,4 \pm $8,1 \mathrm{~kg})$. Ces femelles reparties aléatoirement par lot de trois, ont reçu par lot un régime témoin (RT) et six autres régimes contenant $7,5 \%$ ou $15 \%$ de TA (RTA7,5 et RTA15), de TH (RTH7,5 et RTH15) ou de TJ (RTJ7,5 et RTJ15). Au cours de l'étude, d'une durée de 152 jours, les cochettes sous régime RTJ15 et deux cochettes sous régime RTJ7,5 ont été retirées après respectivement 14 et 21 jours d'alimentation en raison de diarrhées persistantes. La cochette sous régime RTJ7,5 a avorté à 90 jours de gestation. Aucune différence significative n’a été observée sur la taille moyenne des portées (9,2 porcelets). Les poids moyens à la naissance des porcelets variaient entre $1,3 \pm 0,2 \mathrm{~kg}$ (RTA15) et 1,1 $\pm 0,2 \mathrm{~kg}$ (RTH7,5), tandis que les gains moyens quotidiens (GMQ) des sevrons à 28 jours d’âge fluctuaient entre 124 g/j (RTA15) et 164 g/j (RTH7,5). Les GMQ des porcelets sevrés sous régimes RTH7,5 et RTH15 étaient supérieurs à ceux des sevrons soumis aux RTA7,5 et RTA15 $(\mathrm{P}<0,05)$. Ainsi, le TH et le TA pourraient être incorporés à $15 \%$ dans la ration alimentaire des cochettes en reproduction.

Keywords: Porcs, tourteaux, hévéa, jatropha, anacarde, reproduction

\section{Introduction}

En Côte d’Ivoire, le développement de la filière porcine est confronté à plusieurs contraintes dont le coût élevé de l'aliment qui représente aujourd'hui plus de 65 \% des coûts de production d'un porc (Chevalier et al., 2007 ; FIRCA, 2009 ; Lacourt, 2012). Les raisons principales de la cherté de l'aliment porc résident d'une part, dans l'augmentation régulière des prix de certains intrants qui sont importés et d'autre part, dans la concurrence entre les filières animales (Quiniou et al., 2011). Afin de réduire les coûts de production liés à l'alimentation, la mise au point de formules alimentaires économiques intégrant les ressources alimentaires locales nonconventionnelles disponibles et à moindre coût a été envisagée. Les 
ressources utilisées sont notamment la farine d'amandes déclassées d'anacarde chez le porc (Yao et al., 2013), le tourteau d'amandes de Hevea brasiliensis détoxifiées chez les poules pondeuses (Atchibri et al., 2008) et le tourteau de noix de Jatropha curcas chez le porc (Wang et al., 2011; Kouadio et al., 2016). En effet, résidu de l'extraction de l'huile de graines, amandes ou noix, les tourteaux ont une teneur en protéines comprise entre 15-64 \%. Leurs compositions en acides aminés essentiels de leurs protéines sont proches de celle de la farine de soja ou de la farine de poisson à l'exception de la lysine, la méthionine et la cystine (Makkar et al., 1998 ; Devappa et al., 2010). Aussi, vu les quantités de graines d'hévéa (13 300 tonnes/an) (CNRA, 2012), les quantités d'amandes déclassées d'anacarde en Côte d'Ivoire (1 200 tonnes/an) (Sanogo, 2012) et les quantités de tourteau de Jatropha curcas produite au Bénin, Burkina Faso, Mali et Sénégal (pays frontalier nord de la Côte d'Ivoire) (Pallière, 2013 ; Amsallem et Tréboux, 2014), importe-t-il de trouver des solutions simples et durables à leur valorisation.

Certains tourteaux tels que ceux du Jatropha curcas et de l'hévéa, contiennent respectivement des esters de phorbol et l'acide cyanhydrique, qui empêchent leur utilisation comme matière première dans l'alimentation animale (Makkar et al., 1997 ; Atchibri et al., 2008 ; Nesseim et al., 2012). Aussi, afin de lever cette contrainte, des méthodes de réduction à moindre coût des esters de phorbol par fermentation avec la papaye verte (Kouadio et al., 2016) et de l'acide cyanhydrique par la chaleur (Moss et al., 2000), ontelle été mises au point avec plus ou moins de succès. A notre connaissance, l'incorporation de matières non-conventionnelles que sont : les tourteaux d'amandes de Hevea brasiliensis et de Jatropha curcas détoxifiés obtenus après l'application des méthodes à moindre coût et le tourteau d'amandes déclassées d'anacarde, n’ont pas été testés dans l'alimentation des porcs. Cette étude préliminaire vise donc à évaluer les effets de leurs incorporations dans les rations alimentaires sur les paramètres zootechniques des cochettes durant la gestation et la croissance de leurs porcelets au cours de la lactation.

\section{Matériel et méthodes}

\section{Situation du site expérimental}

L'étude a été conduite de janvier 2013 à juin 2013 à la porcherie de la ferme de production de l'Institut National Polytechnique Félix HouphouëtBoigny de Yamoussoukro (INP-HB ; Côte d'Ivoire) (6,5 $5^{\circ}$. ; 5,2 ${ }^{\circ}$ O.). Durant l'essai, la température moyenne et l'humidité relative de la région ont été respectivement de $26 \pm 1{ }^{\circ} \mathrm{C}$ et $83 \%$. La pluviométrie moyenne mensuelle était de $1000 \mathrm{~mm}$. 


\section{Animaux et aliments expérimentaux}

Trois verrats (Sus scrofa) âgés de dix mois, ayant un poids moyen de $80 \pm 2 \mathrm{~kg}$ et 21 cochettes primipares non gravides âgées de six mois et d'un poids de 73,4 $\pm 8,1 \mathrm{~kg}$ ont été achetés chez un même éleveur. Les femelles ont été reparties aléatoirement et de façon homogène par lot de trois dans sept loges de $16 \mathrm{~m}^{2}$ ( $4 \mathrm{~m} \mathrm{x} 4 \mathrm{~m}$ ). Tous les animaux, en bon état sanitaire, étaient issus de croisement (Large white $\mathrm{x}$ Landrace). Six régimes expérimentaux ont été formulés à l'aide de l'outil Solver de Excel dans le logiciel MICROSOFT OFFICE 2010. Les aliments ont été fabriqués dans l'atelier de production d'aliments du Laboratoire de Zootechnie de l'INP-HB. Ce sont RTH7,5 et RTH15 contenant respectivement 7,5 et $15 \%$ de tourteaux détoxifiés des amandes d'hévéa (Hevea brasiliensis) (TH), RTA7,5 et RTA15 contenant respectivement 7,5 et $15 \%$ de tourteaux des déclassées amandes de cajou (Anacardium occidentale) (TA) et RTJ7,5 et RTJ15 contenant respectivement 7,5 et $15 \%$ de tourteaux détoxifiés des amandes de pourghère $(\mathrm{TJ})$. Le régime témoin $(\mathrm{RT})$ contenait toutes les matières premières utilisées à l'exception des TH, TA et TJ (Tableau 1). Les graines d'hévéa et les amandes déclassées d'anacarde ont été respectivement acquises dans les plantations hévéicoles du Sud de la Côte d'Ivoire et à l'unité agro-industrielle du Centre (OLAM Dimbokro). Les graines de Jatropha curcas et les autres matières ont été achetés dans la ville de Yamoussoukro.

Tableau 1 : Composition (\% de poids sec) et analyses bromatologiques des régimes expérimentaux

\begin{tabular}{cccccccc}
\hline & \multicolumn{7}{c}{ Régimes expérimentaux } \\
\cline { 2 - 7 } & RT & RTA7,5 & RTA15 & RTH7,5 & RTH15 & RTJ7,5 & RTJ15 \\
\hline Coût (FCFA/kg) & 196,8 & 195,7 & 187,6 & 191,3 & 191,2 & 186,4 & 194,5 \\
\hline Maïs-grain & 63 & 52 & 49 & 47 & 41 & 45 & 39 \\
Farine de poisson & 4 & 7 & 5 & 4,5 & 3,5 & 4,5 & 4,5 \\
Son de blé & 9,5 & 27,5 & 15 & 20 & 22 & 14 & 14 \\
Tourteau de soja & $\mathbf{7 , 5}$ & $\mathbf{0}$ & $\mathbf{0}$ & $\mathbf{2 , 5}$ & $\mathbf{1}$ & $\mathbf{2}$ & $\mathbf{2}$ \\
Concentré de porc & 2 & 0 & 0 & 1,5 & 1,5 & 1 & 1 \\
Farine basse de riz & 8 & 0 & 10 & 11 & 10 & 20 & 18,5 \\
Coquillage & 0 & 0 & 0 & 0 & 0 & 0 & 0 \\
Tourteau d'anacarde & 0 & 7,5 & $\mathbf{1 5}$ & 0 & 0 & 0 & 0 \\
Tourteau d'hévéa & 0 & 0 & 0 & $\mathbf{7 , 5}$ & $\mathbf{1 5}$ & 0 & 0 \\
Tourteau de Jatropha & 0 & 0 & 0 & 0 & 0 & $\mathbf{7 , 5}$ & $\mathbf{1 5}$ \\
Prémix porc & 1 & 1 & 1 & 1 & 1 & 1 & 1 \\
Sel (NaCl) & 5 & 5 & 5 & 5 & 5 & 5 & 5 \\
\hline Total & 100 & 100 & 100 & 100 & 100 & 100 & 100 \\
\hline MS (\%) & $87,1 \pm 0,1$ & $86,6 \pm 1,0$ & $88,21 \pm 0,03$ & $87,3 \pm 0,1$ & $88,3 \pm 0,1$ & $88,3 \pm 0,1$ & $87,3 \pm 0,2 \mathrm{a}$ \\
MM (\%MS) & $6,0 \pm 0,4$ & $4,2 \pm 0,2$ & $4,34 \pm 0,3$ & $5,4 \pm 0,0$ & $6,9 \pm 0,0$ & $09,1 \pm 0,1$ & $10,0 \pm 0,1$ \\
MO (\%MS) & $94,0 \pm 0,4$ & $95,8 \pm 0,2$ & $95,7 \pm 0,3$ & $94,6 \pm 0,0$ & $93,1 \pm 0,0$ & $90,9 \pm 0,1$ & $90,1 \pm 0,1$ \\
MAT (\%MS) & $17,8 \pm 0,5$ & $17,7 \pm 0,5$ & $17,6 \pm 0,5$ & $17,2 \pm 0,3$ & $17,2 \pm 0,5$ & $15,5 \pm 0,2$ & $14,9 \pm 0,8$ \\
MG (\%MS) & $1,0 \pm 0,12$ & $1,6 \pm 0,0$ & $3,2 \pm 0,7$ & $1,9 \pm 0,1$ & $4,4 \pm 0,3$ & $1,2 \pm 0,2$ & $2,4 \pm 0,0$ \\
CB (\%MS) & $6,1 \pm 1,5$ & $3,3 \pm 1,5$ & $5,5 \pm 3,6$ & $5,7 \pm 2,7$ & $8,1 \pm 1,6$ & $12,7 \pm 1,2$ & $15,7 \pm 0,1$ \\
E M (kcal / kg MS) & 3222,8 & 3569,6 & 3476,6 & 3323,8 & 3189,5 & 2513,5 & 2282,3 \\
HCN (ppm ou mg/kg) & 2,6 & 5,1 & 8,9 & 24,4 & 55,4 & 24,2 & 55,30 \\
\hline & & $* 1 €$ & 655,957 F CFA & & &
\end{tabular}


Les moyennes portant la même lettre sur la ligne sont statistiquement identiques $(\mathrm{P}>0,05)$.

L’énergie métabolisable (EM) a été calculée par la formule de Sibbald (1995) suivante : EM= 3951 + 54,4 MG - 88,7 CB - 40,8 MM

$\mathrm{MS}=$ matière sèche en $\%$ de la matière fraîche, $\mathrm{MM}=$ matière minérale, $\mathrm{MG}=$ matière grasse, $\mathrm{MAT}=$ matières azotées totales, $\mathrm{MO}=$ matière organique, $\mathrm{CB}=$ cellulose brute, $\mathrm{HCN}=$ acide cyanhydrique

\section{Méthodes de production des tourteaux}

Les amandes de graines d'hévéa, de pourghère et d'anacarde sèches ont été broyées avant d'être mélangées de manière homogène à l'eau à raison de deux litres d'eau pour $10 \mathrm{~kg}$ d'amandes broyées. L’homogénat a été placé dans une cuve et couvert par une bâche puis séché pendant deux heures au soleil. Par la suite, l’homogénat séché a été pressé à l’aide d’une presse artisanale « presse à manioc ». Après le recueil de 3 litres d’extrait lipidique pour $10 \mathrm{~kg}$ d'homogénat, le bloc de tourteau résultant a été concassé manuellement puis les mottes ont été séchées à nouveau. L'élimination partielle de l'acide cyanhydrique des mottes de TH a été faite par chauffage au feu de bois. Celle des toxines du TJ a été faite selon la méthode décrite par Kouadio et al. (2016). Les tourteaux détoxifiés ont été séchés à nouveau au soleil pendant une journée et broyés au broyeur à végétaux donnant ainsi un tourteau utilisable comme intrant.

\section{Conduite de l'élevage}

L’essai a duré 152 jours repartis en trois périodes. Il a débuté par une période d'adaptation aux conditions d'élevage de 10 jours avant la période de gestation. Au cours de cette période, tous les animaux ont reçu l'aliment témoin. La période d'accouplement et de gestation de 114 jours a débuté par l'introduction de la femelle au mâle. Durant la phase d'adaptation et la première moitié de la gestation, la ration par animal était de $2 \mathrm{~kg}$ par jour. Elle est passée à $2,5 \mathrm{~kg}$ durant la seconde moitié de la gestation. A l'approche des mises bas, toutes les femelles ont été placées en loge individuelle de maternité jusqu'à la fin de la période de lactation de 28 jours. Au cours de la lactation, les femelles et leurs portées ont reçus $4 \mathrm{~kg}$ d'aliments. Les aliments et l'eau de boisson ont été distribués deux fois par jour. Par ailleurs, les refus d'aliments ont été pesés régulièrement avant chaque nouvelle distribution. Durant l'essai, les animaux à jeun, étaient pesés tous les 15 jours et les performances de reproduction ont été enregistrées à la mise-bas. Les analyses biochimiques ont concerné les analyses bromatologiques (matière sèche analytique, matière minérale, matière azotée totale, matière grasse, fibre et en acide cyanhydrique) des régimes expérimentaux. 


\section{Analyses statistiques}

À la fin de l'expérience, les valeurs moyennes des paramètres d'ingestion, de croissance et de reproduction par régime testé ont été soumises à l'analyse de covariance (ANCOVA) des poids des truies et des porcelets initialement non homogènes ainsi que la taille de la portée. La comparaison multiple des moyennes a été effectuée au seuil de signification de $5 \%$ par le test de Student-Newman-Keuls si les différences révélées par l'ANCOVA étaient significatives. Tous ces calculs ont été effectués avec le logiciel Statistica 7.1.

\section{Résultats}

\section{Production de tourteaux et compositions des régimes expérimentaux}

Pour 100 kg de graines d'hévéa ou de pourghère, respectivement 53 $\mathrm{kg}$ et $62 \mathrm{~kg}$ d'amandes ont été obtenus. Les amandes broyées et humidifiées étaient pressées pour des résidus respectifs de $49 \mathrm{~kg}$ et de $58 \mathrm{~kg}$. Les rendements en $\mathrm{TH}$ et $\mathrm{TJ}$ ont été respectivement de $47 \%$ et de $56 \%$ des graines et respectivement de $88,7 \%$ et de 90,3 \% d'amandes. Le rendement en TA a été de 80,5 \% d’amandes. Les régimes RT, RTA7,5, RTH7,5, RTA15 et RTH15 étaient iso-protéiques avec environ $17 \%$ de MAT contrairement aux régimes RTJ7,5 et RTJ15 dont la valeur moyenne de la teneur en MAT était de $15 \%$ (Tableau 1).

\section{Ingestion et évolution pondérale des cochettes durant la gestation}

Le régime RTJ15 a été arrêté après 14 jours d'alimentation à cause de diarrhées persistantes avec une ingestion moyenne de $1601 \pm 300 \mathrm{~g} / \mathrm{j}$ et une perte de poids moyen de $95 \pm 75 \mathrm{~g} / \mathrm{j}$. Deux des cochettes soumises au régime RTJ7,5 ont été retirées de l'essai après trois semaines d'ingestion pour les mêmes raisons. Au cours de la gestation, aucune différence significative n'a été observée entre les gains moyens quotidiens et entre les indices de consommation $(\mathrm{P}>0,05)$ des cochettes sous les cinq régimes restants. Les valeurs moyennes de l'ingestion alimentaire des cochettes recevant RTA15 ou RTH15 différaient de celles des autres régimes $(\mathrm{P}<0,05)$ (Tableau 2). Quant au régime RTJ7,5, la cochette gestante avait une ingestion moyenne de $1,8 \pm 0,4 \mathrm{~kg} / \mathrm{j}$ pour un gain moyen quotidien de $402 \mathrm{~g} / \mathrm{j}$ durant 90 jours de gestation. Par la suite, elle consommait toujours son régime alimentaire jusqu'à la fin de l'essai, soit plus de 150 jours.

Tableau 2 : Paramètres de croissance et l'ingestion des cochettes pendant la gestation

\begin{tabular}{ccccccc}
\hline & \multicolumn{5}{c}{ Régimes expérimentaux } \\
\cline { 2 - 7 } GMQ (g/j) & RT & RTA7,5 & RTA15 & RTH7,5 & RTH15 & RTJ7,5 \\
\cline { 2 - 7 } IM (g/j) & $2170 \pm 10 \mathrm{~b}$ & $2270 \pm 10 \mathrm{~b}$ & $2330 \pm 30 \mathrm{c}$ & $2240 \pm 0,01 \mathrm{~b}$ & $2260 \pm 0,01 \mathrm{a}$ & $1807 \pm 400$ \\
IC & $3,5 \pm 0,4 \mathrm{a}$ & $3,7 \pm 0,6 \mathrm{a}$ & $4,1 \pm 0,8 \mathrm{a}$ & $3,5 \pm 0,4 \mathrm{a}$ & $3,1 \pm 0,2 \mathrm{a}$ & 4,5 \\
\hline
\end{tabular}


Les moyennes portant la même lettre sur la ligne sont statistiquement identiques $(\mathrm{P}>0,05)$.

$\mathrm{GMQ}=$ gain moyen quotidien, IM= ingestion moyenne, $\mathrm{IC}=$ indice de consommation.

\section{Mises-bas et croissance au cours de l'allaitement}

Les paramètres de reproduction des truies soumises aux différents régimes sont présentés dans le tableau 3. La seule truie gestante sous le régime RTJ7,5 a fait un avortement de cinq cochonnets à 90 jours de gestation. Les avortons présentaient tous des érythèmes sur la peau (Photo 1). La taille moyenne de portée était de 9,2 $\pm 0,5$. Les régimes testés n’ont pas influencé les tailles des portées $(\mathrm{P}>0,05)$. Les analyses statistiques révélaient que les poids à la naissance des porcelets des régimes RTA15 (1,3 $\pm 0,2 \mathrm{~kg})$ et RTH7,5 $(1,1 \pm 0,2 \mathrm{~kg})$ qui différaient l'un de l'autre $(\mathrm{P}<0,05)$, étaient supérieurs à ceux des autres régimes $(\mathrm{P}<0,05)(\mathrm{RT}, \mathrm{RTA7,5}$ et RTH15) qui eux, ne différaient pas $(\mathrm{P}>0,05)$. Les GMQ des porcelets soumis aux régimes contenant le TA (RTA7,5 et RTA15) ne différaient pas ( $\mathrm{P}>0,05)$ et étaient inférieurs à ceux des porcelets sous les régimes contenant le $\mathrm{TH}$ (RTH7,5 et RTH15). Le GMQ des porcelets recevant le régime témoin (RT) était similaire à chacun des deux grounes ( $\mathrm{P}>0.0 .5$ ) (Tableau 3).

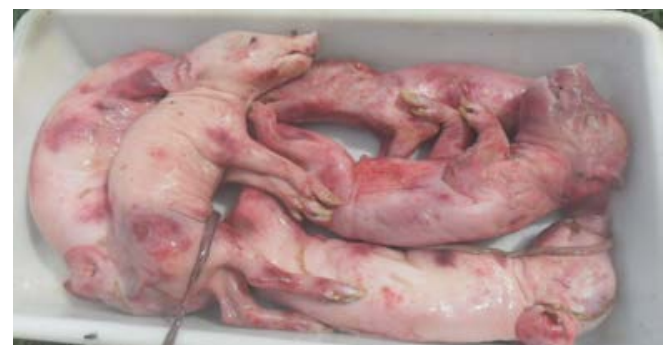

Photo 1 : Avortons du régimes RTJ7,5 Dimension du bac : $25 \mathrm{~cm}$ x $40 \mathrm{~cm}$

Tableau 3 : Paramètres de reproduction étudiés

\begin{tabular}{|c|c|c|c|c|c|}
\hline \multirow{2}{*}{ Paramètres de reproduction } & \multicolumn{5}{|c|}{ Régimes expérimentaux } \\
\hline & RT & RTA7,5 & RTA15 & RTH7,5 & RTH15 \\
\hline Nombre de truies mises en lutte & 3 & 3 & 3 & 3 & 3 \\
\hline Nombre de truie ayant mis bas & 3 & 3 & 2 & 3 & 2 \\
\hline Nombre de porcelets vivants & 29 & 29 & 18 & 26 & 18 \\
\hline Taille moyenne de portée & $9,7 \pm 0,6 a$ & $9,7 \pm 1,5 a$ & $9,0 \pm 4,2 \mathrm{a}$ & $8,7 \pm 0,6 a$ & $9,0 \pm 1,0 \mathrm{a}$ \\
\hline Poids à la naissance (kg) & $1,2 \pm 0,1 \mathrm{ab}$ & $1,3 \pm 0,2 \mathrm{ab}$ & $1,3 \pm 0,2 b$ & $1,1 \pm 0,2 \mathrm{c}$ & $1,3 \pm 0,2 \mathrm{ab}$ \\
\hline Nombre de porcelets sevrés & 29 & 28 & 16 & 25 & 17 \\
\hline GMQ au sevrage $(\mathrm{g} / \mathrm{j})$ & $140 \pm 19 a b$ & $128 \pm 37 a$ & $124 \pm 39 a$ & $164 \pm 35 c$ & $151 \pm 41 \mathrm{bc}$ \\
\hline
\end{tabular}

Les moyennes portant la même lettre sur la ligne sont statistiquement identiques $(\mathrm{P}>0,05)$. 


\section{Discussions}

\section{Ingestion alimentaire des cochettes au cours de la gestation}

Au regard des résultats enregistrés, il ressort que la valorisation du tourteau détoxifié des amandes d’hévéa dans l'alimentation des cochettes en gestation est à encourager dans les régions où elles sont disponibles. Le risque de toxicité au cyanure chez les animaux qui ingéraient le tourteau d'Hevea brasiliensis (Nwokolo, 1987) et sa non-utilisation à des fins alimentaires (Yeong et al., 1981) dans l’alimentation animale étaient liés aux teneurs importantes d'acide cyanhydrique résiduelles après les traitements de l'époque. Notre méthode de détoxification a créé les conditions d'une élimination optimale de l'acide cyanhydrique (Atchibri et al., 2008). Cette élimination de l'acide cyanhydrique correspond à un processus biochimique au cours duquel la linamarase, enzyme hydrolysant les glycosides cyanogénétiques, est d'abord activée pour libérer la cyanhydrine (Mallika et Maggis, 1990). La cyanhydrine est ensuite hydrolysée à $\mathrm{pH}$ acide pour donner l'acide cyanhydrique et l'acétone. Enfin, l'acide cyanhydrique est éliminé du milieu par aération et par ventilation (Monekosso, 1976). Les teneurs en acide cyanhydrique des régimes expérimentaux étaient inférieures aux normes en alimentation animale prévues par l'Union Européenne (2,6 à $55,4 \mathrm{mg} / \mathrm{kg}$ contre $100 \mathrm{mg} / \mathrm{kg}$ ) (Amani et al., 2007).

$\mathrm{Au}$ cours de la gestation, les fortes ingestions alimentaires observées avec les régimes contenant $15 \%$ de tourteau détoxifié de graines d'hévéa ou d'amandes d'anacarde pourraient s'expliquer par la corrélation entre l'ingestion alimentaire et la teneur en cellulose brute des régimes (Palisse et al., 1979).

\section{Paramètres de reproduction et de croissance au cours de l'allaitement}

En comparaison aux résultats obtenus par Dovonou (2002) avec des porcs (Large white $\times$ Landrace), la taille moyenne des portées a été nettement améliorée dans l'ensemble des régimes (9 contre 7,9). Par ailleurs, les poids moyens des porcelets enregistrés à la naissance étaient également supérieurs à celui obtenu par Dovonou (2002) (1,0 kg). Selon Gendron et Gillot (2003), une meilleure utilisation des nutriments assimilés pendant la gestation pour la croissance fœtale au détriment du gain de poids pourrait en être la cause.

L’impact positif de l’incorporation du tourteau de graines d’hévéa est remarquable. En effet, les GMQ des sevrons sous RTH15 et RTH7,5 étaient comparables à ceux obtenus par des porcelets de 35 jours (160 g/j) (MIPRARH, 2003). Toutefois, ces GMQ étaient inférieurs à ceux des races exotiques qui variaient entre 200 et $300 \mathrm{~g} / \mathrm{j}$ pour un sevrage réalisé à 28 jours (Quiniou et al., 2011). Cette baisse de GMQ enregistrée chez les porcelets du régime RTH15 par rapport à celui des porcelets du régime RTH7,5 pourrait 
s'expliquer par la corrélation négative existant entre la teneur en matière grasse des régimes et les GMQ des porcelets (Yao et al., 2013). Au regard des résultats, les effets des tourteaux d'hévéa et d'amandes d'anacarde doivent être évalués sur les porcelets en post-sevrage à des taux d'incorporation supérieurs ou égales à $15 \%$ avec les analyses des carcasses afin d'apprécier leurs efficiences sur toute la chaine de production d'un porc charcutier.

\section{Cas du tourteau détoxifié des amandes de Jatropha curcas}

Les diarrhées persistantes observées chez les cochettes nourries au régime RTJ15 pourraient être liées à l'ingestion du tourteau qui malgré le processus de détoxification mis en œuvre semble contenir encore quelques facteurs anti-nutritionnels notamment les esters de phorbol. Des signes cliniques similaires ont été observés chez les moutons et les chèvres ayant reçu des graines de Jatropha curcas à 0,05, 0,5 et $1 \mathrm{~g} / \mathrm{kg} / \mathrm{jour}$ (Ahmed et Adam, 1979). Cependant, la consommation régulière du régime RTJ7,5 par la dernière cochette durant plus de 150 jours demeure une surprise et encourage à apporter quelques améliorations dans la technique à moindre coût de détoxification du tourteau de jatropha notamment avec l'utilisation de la culture symbiotique de bactéries et de levures dans un milieu sucré.

La présence d'érythème sur la peau des avortons de la seule femelle gestante pourrait être due aux esters de phorbol qui agissent de manière préférentielle sur les membranes biologiques (Evans et Edwards, 1987). En effet, les esters de phorbol agissent sur les protéines kinases C (PKC) de la même manière que le diacylglycérol, en lui permettant d'agir dans la médiation de la réponse cellulaire aux stimuli extracellulaires conduisant en particulier à la prolifération et à la différenciation cellulaire ou à l'apoptose (Blumberg et al., 1987). Ces phénomènes se traduisent principalement par une inflammation ou une progression tumorale (Goel et al., 2007). L'apparition de l'érythème ne semble pas due à un effet direct des esters de phorbol sur la peau, mais à la libération, sous l'effet des esters de phorbol, de médiateurs endogènes de l'inflammation au niveau de la micro vascularisation (Nesseim et al., 2012).

\section{Conclusion}

Le tourteau des amandes de graines d’hévéa et celui des amandes de noix de cajou pourraient être incorporés au taux de $15 \%$ dans la ration alimentaire des truies en période de reproduction. Cependant, ces résultats doivent être confirmés avec des effectifs plus élevés. L’utilisation de ces tourteaux en production animale permettrait également de réduire le coût de l'aliment de porc et sa dépendance vis-à-vis de certaines matières premières importées comme les tourteaux de soja. Concernant le tourteau de Jatropha 
curcas, le processus de détoxification biologique doit être poursuivi avec par exemple l'utilisation de la culture symbiotique de bactéries et de levures dans un milieu sucré.

\section{References:}

1. Ahmed, O. M. M., \& Adam, S. E. I. (1979). Toxicity of Jatropha curcas in sheep and goats. Research in Veterinary Science, 27, 89-96.

2. Amani, G., Nindjin, C., N’zué, B., Tschannen, A., \& Aka, D. (2007). Potentialités à la transformation du manioc (Manihot esculenta crantz) en Afrique de l'Ouest. Actes du $1^{\mathrm{er}}$ Atelier International, $\mathrm{p}$ 358.

3. Amsallem I., \& Tréboux M , (2014). Tourteau de Jatropha : perspectives et contraintes pour la valorisation. Rapport JatroREF, p 23.

4. Atchibri, L. O., Atcho, O., Kouakou, B., \& Keli, J. (2008). La graine d'hévéa appauvrie en acide cyanhydrique par la méthode de triple séchage offre un tourteau propre à la consommation de la poule pondeuse. Revue Africaine de Santé et de Productions Animales, 6 (3-4), 195-198.

5. Blumberg, P. M. (1987). Phorbol esters as probes of the modulatory site on protein kinase $\mathrm{C}$ - an overview. Botanical Journal of the Linnean Society, 94(1-2), 283-292.

6. Chevalier, P., \& Halewyn, M.-A. (2007). Prions et farines carnées destinées à l'alimentation porcine : risques pour la santé humaine. Rapport scientifique. Institut National de Santé Publique Quebec.

7. CNRA (2012). Les programmes de recherches 2012-2015. CNRA, Programme hévéa.

8. Devappa, R. K., Makkar, H. P. S., \& Becker, R. K. (2010). Biodegradation of Jatropha curcas phorbol esters in soil. Journal of the Science of Food and Agriculture, 90(12), 2090-2097.

9. Dovonou, M. N. (2002). Performances zootechniques des races porcines au sud Bénin et perspectives d'amélioration par croisement avec le Piétrain stress négatif, Mémoire. DES Gestion des ressources animales et végétales en milieux tropicaux. Liège, Belgique, Université de Liège.

10. Evans, F. J., \& Edwards, M. C. (1987). Activity correlations in the phorbol esters series. Botanical Journal of the Linnean Society, 94 (12), 231-246.

11. FIRCA (2009). La filière porcine. La filière du progrès, 2(4), 1-28.

12. Gendron, E., \& Gillot, A. S. (2003). Fabrication de l'aliment à la ferme en élevage porcin : contribution à la recherche de traceurs de 
l’homogénéité du mélange, Veterinary thesis, Ecole Nationale Vétérinaire Toulouse, Toulouse, France.

13. Goel, G., Makkar, H. P. S., Fancis, G., \& Becker, K. (2007). Phorbol esters: structure, biological activity and toxicity in animals. International Journal Toxicology, 26(4), 279-288.

14. Kouadio, K. B., Dougnon, M. G., \& Kouakou, N. D. V. (2016). Effet de la supplémentation de l'aliment croissance des coquelets (Warren) par du tourteau de Jatropha curcas détoxifié. Journal of Animal \& Plant Sciences 28(3), 4479-4487.

15. Lacourt, E. (2012). Démystifier le coût d'alimentation d'un porc : de la formule à la ferme. Agri-nouv. Sci, 32 (1), 58-61.

16. Makkar, H. P. S., Becker, K., Sporer, F., \& Wink, M. (1997). Studies on nutritive potential and toxic constituents of different provenances of Jatropha curcas. Journal of Agricultural and Food Chemistry, 45, 3152-3157.

17. Makkar, H. P. S., Aderibigde, A. O., \& Becker, K. (1998). Comparative evaluation of non-toxic and toxic varieties of Jatropha curcas for chemical composition, digestibility, protein degradability and toxic factors. Food Chemistry, 62(2), 207-215.

18. Mallika, G. V., \& Maggis, E. (1990). Studies on controlling the action of lipase and linamarase during rubber kernel processing. Journal of the National Science Council of Sri Lanka 19 (2), 99-106.

19. MIPRARH, 2003. Rapport national sur l'état des ressources zoogénétiques. Ministère de la Production Animale et des Ressources Halieutiques, Abidjan, Côte d'Ivoire.

20. Monekosso, E. G. C. (1976). Cyanideneys: In Garter's Veterinary Toxicology $3^{\text {rd }}$ Ed. Baillie Tindall and Cassell London.

21. Mosso, K., Bohoua, G., \& Gnankri, D. (2000). Détoxication en cyanure des aliments dérivés du manioc. Industries alimentaires et agricoles, 117(10), 41-42.

22. Nesseim, T. D. T., Fillet, M., Mergeai, G., Dieng, A., \& Hornick, J.L. (2012). Principes toxiques, toxicité et technologie de détoxification de la graine de Jatropha curcas L. (Synthèse bibliographique). Biotechnologie, Agronomie, Société et Environnement 16(4), 531-540.

23. Nwokolo, E. (1987). Biochemical and nutritional qualities of rubber seed meal. Tropical Agriculture, 64(3), 170-171.

24. Palisse, M., Colin, M., \& Maury, Y. (1979). Etude de quelques aspects du transit digestif chez la truie en gestation : variation avec le taux de cellulose et relation avec le phénomène de constipation. Journées Recherche Porcine en France, 4, 217-222. 
25. Pallière B. (2013). Altlas de la production d'huile de Jatropha au Bénin, Burkina-Faso, Mali et au Sénégal. Pôle « Production d’huile de Jatropha ». Rapport JatroREF, p 10.

26. Quiniou, N., Primot, Y., Peyronnet, C., \& Quinsac, A. (2011). Des aliments porcs moins riches en protéines et formulés à base de tourteau de colza et d'acides aminés de synthèse, dont la L -Valine, permettent de réduire le recours au tourteau de soja. Journées Recherche Porcine en France, 43, 135-136.

27. Sanogo, M. (2012). Industrialisation de la filière anacarde en Côte d'Ivoire. $7^{\text {th }}$ Annual Conference of Africa Cashew Alliance\# Visited 18 septembre 2016, from http://www.africancashewalliance.com/sites/default/files/malamine_s anogo_-_areca_-_aca_conference_19_sept_2012.pdf

28. STATA (2012). Stata/IC 12.0 for windows. Lakeway Drive College Station.

29. Wang, H., Chen, Y., Zhao, Y. N., Liu, H., Liu, J., Makkar, H. P. S., \& Becker, K. (2011). Effects of replacing soybean meal by detoxified Jatropha curcas kernel meal in the diet of growing pigs on their growth, serum biochemical parameters and visceral organs. Animal Feed Science and Technology, 170, 141-146.

30. Yao, K. S. A., Kimsé, M., Soro, D., \& Fantodji, A. (2013). Effet de l'incorporation de la noix de cajou dans les rations alimentaires sur les performances de croissance des porcs : phases post-sevrage et de croissance. International Journal of Biological and Chemical Sciences, 7(2), 479-488.

31. Yeong, S. W., Seyd-Ali, A. B., \& Yusof, N. (1981). The use of rubber seed meal in poultry. The effect of rubber seed meal in layer diets. Malaysian Agricultural Research and Development Institute (MARDI) Research Bulletin, 9 (1), 92-96. 